\title{
Towards a High Diffraction Efficiency of Photorefractive Multiple Quantum Wells
}

\author{
T. Z. Ward, P. Yu, S. Balasubramanian, M. Chandrasekhar, H.R. Chandrasekhar \\ Department of Physics and Astronomy, University of Missouri-Columbia, Columbia, Missouri 65211
}

\begin{abstract}
We propose a method to improve the diffraction efficiency of photorefractive multiple quantum well devices in the transverse-field geometry. Higher efficiencies have been achieved through systematic electrical modulation studies.
\end{abstract}

\section{INTRODUCTION}

Photorefractive quantum well (PRQW) devices have shown promise in laser based ultrasound detection and in vivo biomedical imaging [1-4]. In these applications, the device records dynamic holograms using adaptive interferometry. The device acts as a holographic beam combiner in two-wave and four-wave mixing configurations. The sensitivity of the system is determined by the minimum detectable intensity and diffraction efficiency [5]. A relatively low diffractive efficiency of the PRQW device reduces the system sensitivity. For example, in a coherent domain biomedical imaging system, a sensitivity of $-80 \mathrm{~dB}$ has been measured for AlGaAs/GaAs PRQW devices. This gives a limited image depth in comparsion with conventional optical coherence tomography which shows a sensitivity of $-110 \mathrm{~dB}$, about $30 \mathrm{~dB}$ higher than a semiconductor based system. Although we can increase the reference intensity to gain more diffraction efficiency for the PRQW device in a wave-mixing setup, the main limitation in diffraction efficiency is the total intensity of the writing beams on the semiconductor devices. Because the devices operate under high applied electric fields and the laser intensities generate high photoconductivity, the devices experience Joule heating. The Joule heating must be kept below a safe value, else thermal run-away occurs, and the device may be destroyed.

When a laser and an electric field are applied to the PRQW device, the Joule heating is given by:

$$
\mathrm{P}_{J}=\frac{I_{J}}{h v} \alpha \mu \tau e E^{2} H d w
$$

where $h v$ is the incident photon energy, $\alpha$ is the absorption coefficient, $\mu \tau$ is the mobility-lifetime product of the dominant carrier, $e$ is the electron charge, $I_{J}$ is the incident intensity, and $E$ is the applied electric field. The window dimensions included in the Joule heating are given as follows: $H$ is the window height, $w$ is the window width and $d$ is the PRQW thickness. The critical heating depends on the electrical power per area. For PRQW devices fabricated with epoxy on glass, the maximum allowable electrical power density is $\mathrm{P}_{\mathrm{J}}=100$ $\mathrm{mW} / \mathrm{cm}^{2}$.

Previously, higher Joule intensities could be achieved by working at a lower field or with shorter carrier lifetimes. Controlling the lifetime (and possibly the mobility) is a more practical means of increasing the Joule intensity. However, these procedures are limited by the conditions in device fabrication and only give limited improvement. To overcome the Joule heating problem and increase the diffraction efficiency, we used a low duty cycle pulsed electric field on the $\mathrm{AlGaAs} / \mathrm{GaAs}$ devices.

\section{EXPERIMENTS}

We used standard techniques to measure Joule heating and differential transmission. A resistor of 
1.03 $\mathrm{M} \Omega$ was connected in series to the device. A pulsed electric field was applied across the device window. The device was illuminated by a He-Ne laser. A diode laser (HL832SE, Hitachi) was used in twowave mixing experiments. The wavelength of the laser was tuned by temperature. The signal beam interfered with the reference beam at the PRQW with an intensity ratio of $1: 3$ and a fringe period of $30 \mu \mathrm{m}$. A Si detector (Det110, Thorlabs) with a 750nm long pass filter and a lock-in amplifier (SR510, Stanford Research) were used in two-wave mixing experiments.

\section{RESULTS AND DISCUSSION}

Figure 1 shows Joule heating versus duty cycle under different applied electric fields. The laser intensity was kept at $17 \mathrm{~mW} / \mathrm{cm}^{2}$. We also tested other intensities, and the results were comparable. Since the electric field was applied parallel to the quantum well layers (transverse or Franz-Keldysh geometry), the exciton broadened and changed the electroabsorption. Theoretically, if we only considered the lowest order of the electric field effect, the change in absorption should have been quadratic with the applied field. We observed a deviation from the quadratic function at an electric field of about $5.0 \mathrm{kV} / \mathrm{cm}$, which can be explained as a high order effect.

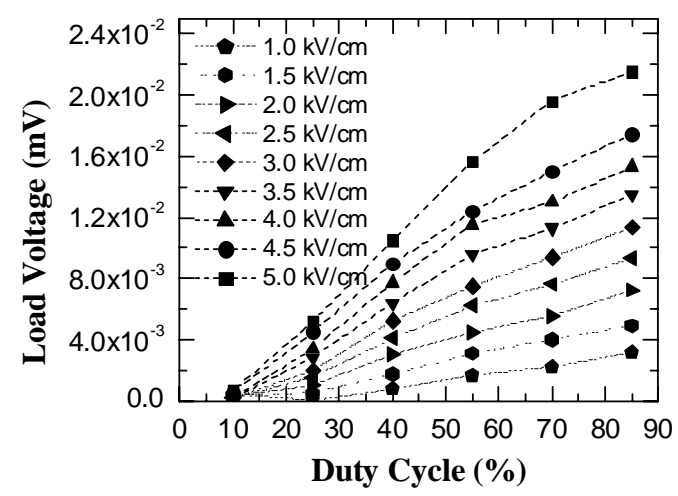

FIGURE 1. Load voltage as a function of duty cycle. Lines are for guidance.

In most wave-mixing applications of the PRQW devices, the relative intensity ratio between the signal and reference should be considered in addition to the total intensity; all should be optimized to obtain higher diffractive efficiencies. Figure 2 shows two-wave mixing as a function of duty cycle. The intensity ratio of $1: 3$ between the signal and reference was selected to ensure high differential transmission and two-beam coupling. As we expected, the average two-wave mixing signal decreased at lower duty cycles. However, the peak value remained nearly the same, even showing a slight increase at the lowest duty cycle. Also at higher duty cycles (about 60\%), saturation of the two-wave mixing signal has been observed to correspond with Joule heating measurements. An estimate of a factor of 17 in diffraction efficiency improvement was observed by using this method when the same Joule heating value was maintained.

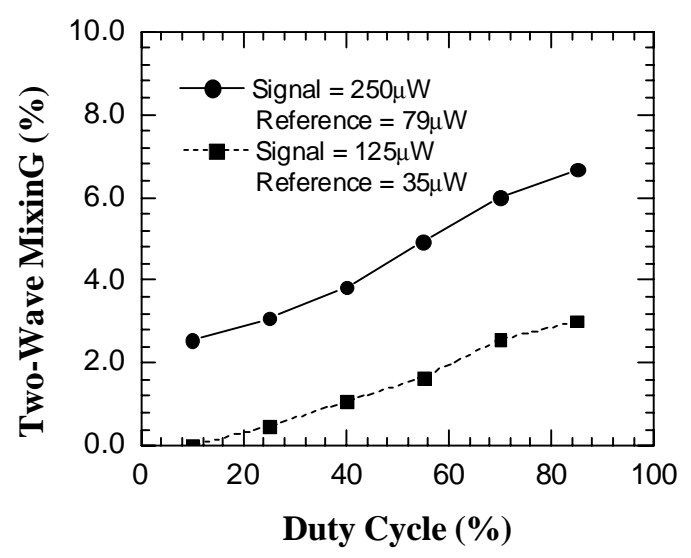

FIGURE 2. Two wave mixing as a function of duty cycle. Beam diameter $2 \mathrm{~mm}$ each. Lines are for guidance.

In conclusion, we tested the concept of using a low duty cycle electric field to ease Joule heating constraints on PRQW devices. Increased diffraction efficiency has been observed, which allow using this method in wave-mixing applications.

\section{REFERENCES}

1. R. Jones, S. C. W. Hyde, M. J. Lynn, N .P. Barry, J. C. Dainty, P. M. W. French, K. M. Kwolek, D. D. Nolte, and M. R. Melloch, Appl. Phys. Lett. 69, 1837-1839 (1996).

2. P. Yu, M. Mustata, P. M. W. French, J. J. Turek, M. R. Melloch, and D. D. Nolte, Appl. Phys. Lett. vol. 83, 575, (2003).

3. P. Yu, L. Peng, D. D. Nolte, and M. R. Melloch, Optics Letters, 28, 819, (2003).

4. P. Yu, L. Peng, M. Mustata, J. J. Turek, M. R. Melloch, and D. D. Nolte, Optics Letters, 29, 68, (2004).

5. D. D. Nolte, T. Cubel, L. J. Pyrak-Nolte, and M. R. Melloch, J. Opt. Soc. Am. B 18, 195-205 (2001). 